\title{
miR-497 inhibits the proliferation and migration of A549 non-small-cell lung cancer cells by targeting FGFR1
}

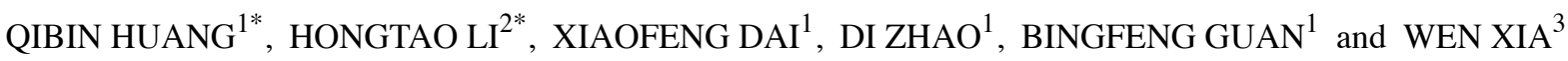 \\ Departments of ${ }^{1}$ Cardiothoracic Surgery, ${ }^{2}$ Oncology and ${ }^{3}$ Anesthesiology, \\ Jingzhou First People's Hospital, Jingzhou, Hubei 434000, P.R. China
}

Received July 15, 2018; Accepted November 14, 2018

DOI: $10.3892 / \mathrm{mmr} .2019 .10611$

\begin{abstract}
Fibroblast growth factor receptor 1 (FGFR1) signaling has been reported to contribute to the carcinogenic progression of various cancer types. Previous studies have demonstrated that FGFR1 expression is increased in non-small cell lung cancer (NSCLC) and promotes cancer cell metastasis. However, the molecular mechanisms underlying increased FGFR1 expression in NSCLC remains largely unknown. In the current study, microRNA (miR)-497 levels were observed to be inversely correlated with FGFR1 expression in tumor samples from patients with NSCLC. In the NSCLC cell line A549, miR-497 overexpression inhibited cell proliferation and migration. Increased expression of miR-497 led to a reduction in FGFR1 expression, at the mRNA and protein levels. In addition, transfection of miR-497 mimics inactivated the protein kinase B (AKT) and c-Jun N-terminal kinase (JNK) signaling pathways, as reduced matrix metallopeptidase 26 expression; all of which are regulated by FGFR1. Using TargetScan software, FGFR1 was also identified as a predicted target gene of miR-497, and a dual luciferase reporter assay confirmed that miR-497 directly regulated FGFR1. Transfection of a recombinant FGFR1 overexpression vector reversed miR-497 mimic-induced arrest of cell growth and migration in A549 cells. In conclusion, the results of the present study identified miR-497 as a potential tumor suppressor gene in NSCLC that may function via repressing FGFR1 expression, and AKT and JNK signaling.
\end{abstract}

Correspondence to: Dr Hongtao Li, Department of Oncology, Jingzhou First People's Hospital, 8 Hangkong Road, Jingzhou, Hubei 434000, P.R. China

E-mail: lihongtaojzfph@sina.com

${ }^{*}$ Contributed equally

Key words: microRNA-497, non-small-cell lung cancer, fibroblast growth factor receptor 1

\section{Introduction}

Lung cancer is the most common cancer worldwide and is the leading cause of cancer-associated mortality despite improvements in diagnosis and therapy (1). More than 1 million mortalities are attributable to lung cancer each year and $<10 \%$ of patients with the disease survive $>5$ years after diagnosis (2). Lung cancer can be divided into two main groups: Small-cell lung cancer (SCLC) and non-SCLC (NSCLC) (3). NSCLC accounts for $75 \%$ of all lung cancers and, despite advances in treatment, the prognosis of patients with NSCLC remains poor, with a 5-year overall survival rate of only $15 \%$ (4). Therefore, it is vital to investigate the molecular mechanisms involved in lung carcinogenesis, and to identify diagnostic and prognostic markers for the early detection and targeted treatment of lung cancer.

Fibroblast growth factor receptor 1 (FGFR1) is a member of the FGFR receptor tyrosine kinase family, consisting of FGFR1, 2, 3 and 4 (5). FGFR proteins interact with fibroblast growth factor to initiate a cascade of downstream signaling pathways that ultimately regulate cell proliferation, survival, migration and differentiation (6). In particular, increased expression and sustained activation of FGFR1 has been reported in several human cancers, including oral and tongue squamous cell carcinoma (7), esophageal squamous cell carcinoma (8) and breast cancer (9). Increased expression of FGFR1 has been observed in $10-20 \%$ of patients with lung cancer, and is correlated with the level of cigarette smoking and poor clinical outcomes in patients with resected smoking-associated squamous cell lung cancer (10). FGFR1 has been identified as a promising molecular target for the treatment of NSCLC, thus providing a novel therapeutic target for these tumors (11). Recently, $\mathrm{Pu}$ et al (12) reported increased expression levels of FGFR1 are associated with a subgroup of NSCLCs, and FGFR1 was identified as an independent prognostic factor associated with poor prognosis, particularly in early stage disease.

MicroRNAs (miRNAs/miRs) are a group of small, noncoding, endogenous single-stranded RNAs that regulate the expression of $\sim 60 \%$ of human genes $(13,14)$. miRNAs reduce gene expression by binding to complementary regions of target mRNA sequences and either inhibit their translation or mediate their degradation via the RNA-induced silencing complex (15). The aberrant expression of miRNAs leads to 
the altered expression of their target genes, which has been demonstrated to contribute to cancer initiation and progression (16-18). In addition to gene amplification, several miRNAs have been reported to directly or indirectly control the expression of FGFRs to mediate cancer development (19-21). In NSCLC, next generation sequencing and microarray-based analyses have revealed the role of several miRNAs in lung carcinogenesis $(22,23)$. However, how these differentially expressed miRNAs promote cancer progression remains elusive.

In the present study, miR-497 expression was observed to be decreased in tumor tissues derived from patients with NSCLC, and was negatively correlated with FGFR1 mRNA levels. Overexpression of miR-497 led to a reduction in the growth and migration of A549 cells, and reduction in FGFR1 at the mRNA and protein levels. FGFR1 was predicted and validated to be a target gene of miR-497. Through regulation of FGFR1, miR-497 inactivated the protein kinase B (AKT) and c-Jun N-terminal kinase (JNK) signaling pathways and repressed matrix metallopeptidase 26 (MMP26) expression. Transfection of recombinant FGFR1 reversed the miR-497 mimics-induced inhibition of A549 cell growth and migration. The results of the present study suggest that miR-497 may exert a tumor suppressive role in NSCLC by binding to FGFR1 mRNA.

\section{Materials and methods}

Tissues. A total of 20 matched NSCLC tumor tissues and normal lung tissues were collected from patients with NSCLC (aged 62.65 \pm 8.92 years old, male:female $=13: 7$ ) admitted to Jingzhou First People's Hospital (Jingzhou, China) between February 2015 and May 2016. All participants provided written consent prior to the study. The experiments were conducted under the supervision of the Jingzhou First People's Hospital Ethics Committee. Following surgery, the tissue samples were immediately stored at $-80^{\circ} \mathrm{C}$ prior to RNA extraction.

Cell lines. The 293 cell line, human BEAS-2B normal lung epithelial cells and the human A549 NSCLC cell line were purchased from the American Type Culture Collection (Manassas, VA, USA), and used within 6 months of receipt. 293 cells were cultured in RPMI-1640 medium (Gibco; Thermo Fisher Scientific, Inc, Waltham, MA, USA) and A549 cells were cultured in Dulbecco's modified Eagle's medium (Gibco; Thermo Fisher Scientific, Inc.). All of the above cell culture media was supplemented with $10 \%$ fetal bovine serum (FBS; Gibco; Thermo Fisher Scientific, Inc.), and cells were maintained in a humidified incubator at $37^{\circ} \mathrm{C}$ with $5 \% \mathrm{CO}_{2}$. BEAS-2B normal lung epithelial cells were cultured in LHC-8 medium (Gibco; Thermo Fisher Scientific, Inc.) and maintained in a humidified incubator at $37^{\circ} \mathrm{C}$ with $5 \% \mathrm{CO}_{2}$.

Cell proliferation assay. The growth rate of A549 cells was analyzed using Cell Counting Kit-8 (CCK-8; Dojindo Molecular Technologies, Inc., Kumamoto, Japan) according to the manufacturer's protocol. Briefly, cells $\left(1 \times 10^{3}\right.$ cells/well $)$ were seeded in 96-well plates and transfected with $50 \mathrm{nM}$ miR-negative control (NC, 5'-UAACCACUUUCACAU
GGUCCUA-3') or miR-497 mimics (5'-CAGCAGCACACU GUGGUUUGU-3') using Lipofectamine ${ }^{\circledR} 2000$ (Invitrogen; Thermo Fisher Scientific, Inc.). At 0, 24, 48 and $72 \mathrm{~h}$ following transfection, $10 \mu \mathrm{l}$ CCK-8 solution was added into the indicated wells and the cells were incubated for a further $1 \mathrm{~h}$. The medium containing CCK-8 was then transferred to a fresh 96-well plate and the absorbance of each well was measured using a microplate reader at $450 \mathrm{~nm}$ (Bio-Rad Laboratories, Inc., Hercules, CA, USA) to determine the cell number.

Cell migration assay. The migration ability of A549 cells was analyzed using a wound healing assay. Briefly, the cells were cultured in a 6-well plate, transfected with miR-NC or miR-497 mimics and cultured until $90 \%$ confluency was reached. A $10 \mu \mathrm{l}$ pipette was then used to scrape the surface monolayer of cells. The cells were subsequently washed with PBS, followed by the addition of medium containing $1 \% \mathrm{FBS}$. Images were captured using a Nikon TE2000 microscope (Nikon Corporation, Tokyo, Japan) at 0 and $30 \mathrm{~h}$ following generation of the scratch-wound. The relative migration area was determined using a light microscope (SZX16-3111; Olympus Corporation, Tokyo, Japan).

RNA extraction and reverse transcription-quantitative polymerase chain reaction $(R T-q P C R)$. At $24 \mathrm{~h}$ after transfection, RNA from tissues and cells was extracted using TRIzol reagent (Invitrogen; Thermo Fisher Scientific, Inc.). For the detection of miRNA, RNA was first reverse transcribed using the PrimeScript RT reagent kit (Takara Biotechnology Co., Ltd.) at $37^{\circ} \mathrm{C}$ for $15 \mathrm{~min}$ followed by incubation at $85^{\circ} \mathrm{C}$ for $5 \mathrm{sec}$. qPCR was then performed to detect miR-497 levels in cells and tissues using the SYBR Premix Ex Taq (Takara Biotechnology, Co., Ltd.). The thermocycling conditions were: Initial denaturation at $95^{\circ} \mathrm{C}$ for $30 \mathrm{sec}$, followed by 40 cycles of $95^{\circ} \mathrm{C}$ for $5 \mathrm{sec}$ and $60^{\circ} \mathrm{C}$ for 30 sec. U6 was used as an internal control for the normalization of miRNA. The primer sequences used to detect U6 and miR-497 were as listed: U6 forward: 5'-TGCGGGTGCTCG CTTCGCAGC-3'; reverse: 5'-CCAGTGCAGGGTCCGAGG T-3'; miR-497 forward: 5'-TGGTGTGAATGATAGGTT ATTTTATT-3'; reverse: 5'-TCCATCTCTCTAAATCCC TACAAAA-3'. For the detection of mRNA expression, RNA was first reverse transcribed into first-strand cDNA using the PrimeScript RT reagent Kit (Takara Biotechnology, Co., Ltd.) at $37^{\circ} \mathrm{C}$ for $15 \mathrm{~min}$ followed by incubation at $85^{\circ} \mathrm{C}$ for $5 \mathrm{sec}$, followed by RT-PCR using SYBR Premix Ex Taq (Takara Biotechnology, Co., Ltd.). The thermocycling conditions were: Initial denaturation at $95^{\circ} \mathrm{C}$ for $30 \mathrm{sec}$, followed by 40 cycles of $95^{\circ} \mathrm{C}$ for $5 \mathrm{sec}$ and $60^{\circ} \mathrm{C}$ for $30 \mathrm{sec}$. Gene expression was quantified using the $2^{-\Delta \Delta \mathrm{Cq}}$ method (24). $\beta$-actin served as an internal control for mRNA expression. The primer sequences used to detect $\beta$-actin and FGFR1 were: $\beta$-actin forward: 5'-CTCCATCCTGGCCTC GCTGT-3'; reverse: 5'-GCTGTCACCTTCACCGTTCC-3'; FGFR1 forward: 5'-GCTAGGTGCCGAGGGTGTT-3'; reverse: 5'-ACTGCAGGCTCCTTCAGAAC-3'.

Western blotting. At $30 \mathrm{~h}$ after cell transfection, protein lysates were prepared using radioimmunoprecipitation assay lysis buffer (Sigma-Aldrich; Merck KGaA, Darmstadt, 
Germany) according to manufacturer's instructions. The western blot was performed using standard procedures. Firstly, protein concentration was quantified by the BCA method. Proteins (15 $\mu \mathrm{g} /$ lane) were separated by $8-10 \%$ SDS-PAGE, transferred to a polyvinylidene difluoride membrane and then blocked with $5 \%$ non-fat milk at room temperature for $2 \mathrm{~h}$. The membranes were then incubated with primary antibodies at $4^{\circ} \mathrm{C}$ overnight, washed with PBS and then incubated with the appropriate secondary antibodies at room temperature for $1 \mathrm{~h}$. Antibodies against FGFR1 [rabbit monoclonal antibody (mAb); cat. no. 9740; 1:1,000], AKT (rabbit mAb; cat. no. 4691; 1:1,000), phosphorylated (p)-AKT (rabbit mAb; cat. no. 13038; 1:1,000), JNK (rabbit mAb; cat. no. 9252; 1:1,000), p-JNK (rabbit mAb; cat. no. 4668; 1:1,000) were purchased from Cell Signaling Technology, Inc. (Danvers, MA, USA), while the $\beta$-actin antibody (mouse mAb; cat. no. A1978; 1:2,000) was purchased from Sigma-Aldrich (Merck KGaA). Secondary antibodies against mouse (cat. no. sc2005; 1:500) and rabbit (cat. no. sc2004; 1:500) were purchased from Santa Cruz Biotechnology, Inc. (Dallas, TX, USA). Finally, the membranes were developed using enhanced chemiluminescence western blot substrate (Vazyme, Piscataway, NJ, USA) and images were captured using an ImageQuant 400 (GE Healthcare, Chicago, IL, USA). Relative band intensity was quantified with ImageJ software (version. 1.8.0; National Institutes of Health).

Dual relative luciferase activity assay. The binding of miR-497 to the 3'-untranslated region (UTR) of FGFR1 was predicted by TargetScan release 7.1 (http://www.targetscan. org/vert_71/) and validated using a dual luciferase reporter assay. The 3'-UTR of FGFR1 mRNA was amplified from cDNA obtained from 293 cells and BEAS-2B cells and annealed into the pGL3-basic vector (Promega Corporation, Madison, WI, USA) between KpnI and Xhol restriction enzyme sites. Two site mutations were introduced into the pGL3-FGFR1 3'-UTR-wild-type (WT) sequence to generate the pGL3-FGFR1 3'-UTR-Mutant using a Quick Site-directed mutation kit (Agilent Technologies, Inc., Santa Clara, CA, USA). 293 cells $\left(1 \times 10^{5}\right)$ and A549 cells $\left(1 \times 10^{5}\right)$ were seeded into $24-w e l l$ plates, and co-transfected with pGL3-FGFR1 3'-UTR-WT (0.4 mg) or pGL3-FGFR1 3'UTR-Mutant $(0.4 \mathrm{mg})$ together with miR-NC $(20 \mathrm{~nm})$ or miR-497 mimics $(20 \mathrm{~nm})$ by Lipofectamine ${ }^{\circledR} 2000$ (Invitrogen; Thermo Fisher Scientific, Inc.), respectively. At $48 \mathrm{~h}$ after transfection, the relative luciferase activity of each group was measured using a dual luciferase reporter system kit normalized to Renilla luciferase activity (Promega Corporation).

Overexpression of FGFR1. The full length open reading frame of FGFR1 was amplified from 293 cell cDNA digested with HindIII and XhoI (New England Biolabs, Inc.) and ligated into the pcDNA3 plasmid (Invitrogen; Thermo Fisher Scientific, Inc.). The primer sequences for FGFR1 were: Forward, 5'-AAG CTTATGTGGAGCTGGAAGTG-3'; reverse, 5'-CTCGAG GGAGGGCGTGTGGGTG-3'. For FGFR1 overexpression, $2 \mu \mathrm{g}$ pcDNA3-FGFR1 was mixed with Lipofectamine ${ }^{\circledR} 2000$ (Invitrogen; Thermo Fisher Scientific, Inc.) and added to the
$2 \times 10^{6}$ cells in cell culture medium. At $8 \mathrm{~h}$ after transfection, the medium was replaced with fresh medium and the cells were maintained for a further $48 \mathrm{~h}$ prior to western blotting analysis.

Statistical analysis. Each experiment was repeated $\geq 3$ times in the present study. Statistical analysis was performed using the GraphPad Prism 7.0 software program (GraphPad Software, Inc., La Jolla, CA, USA). The Pearson correlation test was performed to determine the association between miR-497 and FGFR1 mRNA expression levels. Differences between two groups were analyzed using the Student's t-test. For comparisons among three groups, one-way analysis of variance was performed followed by Newman-Keuls test. All P-values obtained in this study were two-tailed. $\mathrm{P}<0.05$ was considered to indicate a statistically significant difference.

\section{Results}

miR-497 is negatively correlated with FGFR1 in NSCLC tumor tissues. To investigate the expression of miR-497 in NSCLC tumor tissues, RT-qPCR was performed to compare miR-497 levels in tumor and matched normal tissues from 20 NSCLC patients. miR-497 expression was significantly downregulated in tumor tissues compared with matched normal tissues (Fig. 1A). In addition, FGFR1 mRNA levels were increased in tumor tissues compared with matched normal tissues (Fig. 1B). A negative correlation between miR-497 and FGFR1 mRNA levels was observed in NSCLC tumor tissues (Fig. 1C).

Overexpression of miR-497 inhibits the proliferation and migration of NSCLC cells. To investigate the function of miR-497 in NSCLC cells, miR-497 was overexpressed in A549 cells via transfection of miR-497 mimics (Fig. 2A). Overexpression of miR-497 inhibited the proliferative ability of A549 cells (Fig. 2B). Additionally, miR-497 mimics suppressed the migration of A549 cells (Fig. 2C and D).

miR-497 represses FGFR1 expression and inactivates FGFR1-regulated signaling pathways in A549 cells. Subsequent experiments sought to investigate the mechanisms underlying the observed effects of miR-497 overexpression in NSCLC cells. RT-qPCR analysis demonstrated that miR-497 overexpression led to a significant reduction in FGFR1 mRNA levels in A549 cells (Fig. 3A). In addition, western blot analysis revealed that FGFR1 protein levels were downregulated following miR-497 overexpression (Fig. 3B and C). It has been previously reported that FGFR1 activates the phosphoinositide 3-kinase (PI3K)-AKT and JNK signaling pathways promote NSCLC progression $(25,26)$. In the present study, miR-497 mimics reduced p-AKT and p-JNK levels, but not those of AKT or JNK, via regulation of FGFR1, which suggested that PI3K-AKT and JNK signaling pathway activities were repressed following miR-497 overexpression in A549 cells (Fig. 3D and E). MMP26 is an oncogene and is tightly controlled by FGFR1/JNK signaling in NSCLC cells (27). Overexpression of miR-497 in A549 cells decreased MMP26 protein expression in the current study (Fig. 3F and G). 
A

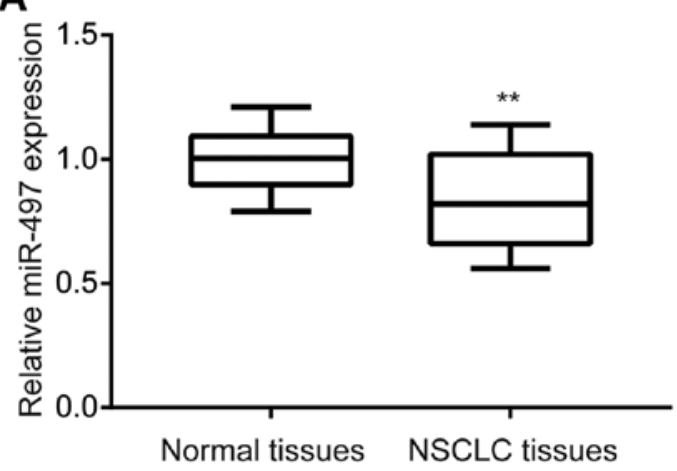

C

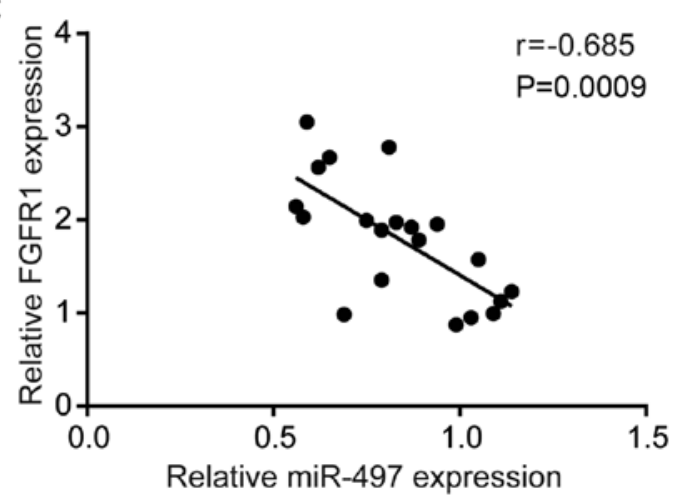

B

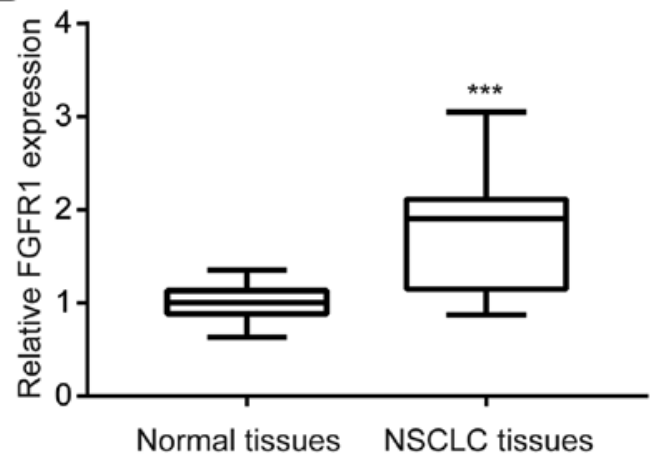

Figure 1. miR-497 is downregulated and inversely correlated with FGFR1 mRNA levels in NSCLC compared with matched normal tissues. (A) miR-497 levels were decreased in tumor tissues from 20 NSCLC patients, whereas (B) FGFR1 mRNA levels were elevated. (C) miR-497 levels were inversely associated with FGFR1 mRNA levels in NSCLC tumor tissues. The experiment was repeated $\geq 3$ times. ${ }^{* *} \mathrm{P}<0.01,{ }^{* * *} \mathrm{P}<0.0001$ vs. normal tissues. miR-497, microRNA-497; NSCLC, non-small cell lung cancer; FGFR1, fibroblast growth factor receptor 1.

A

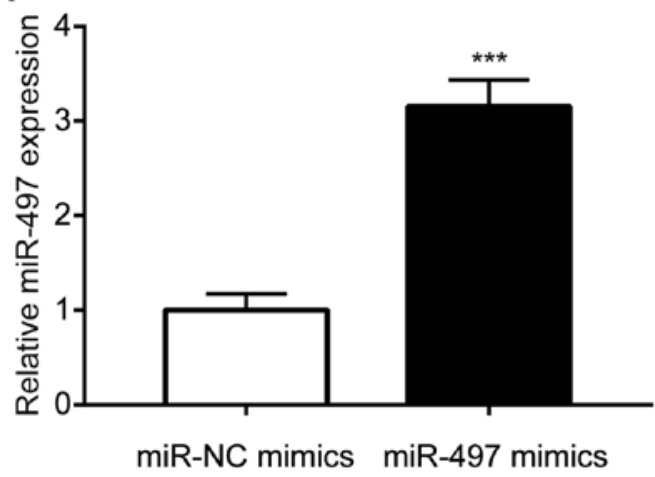

C

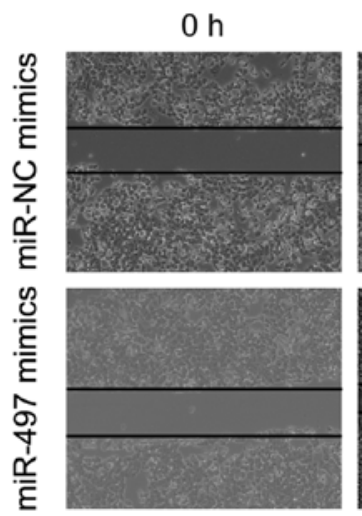

B

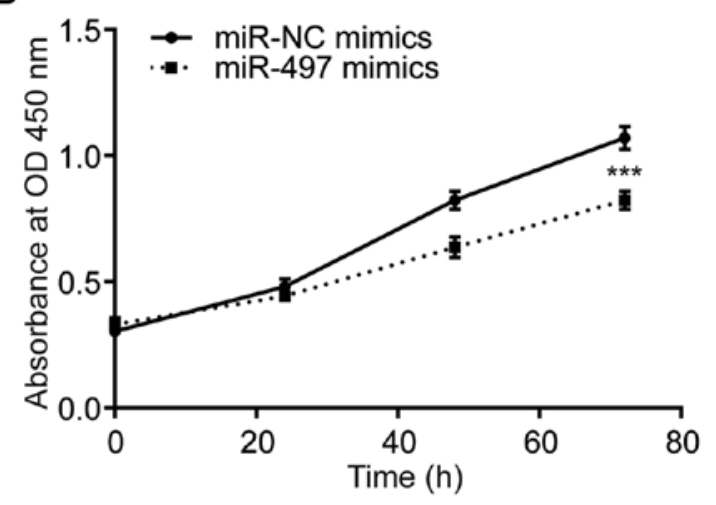

D

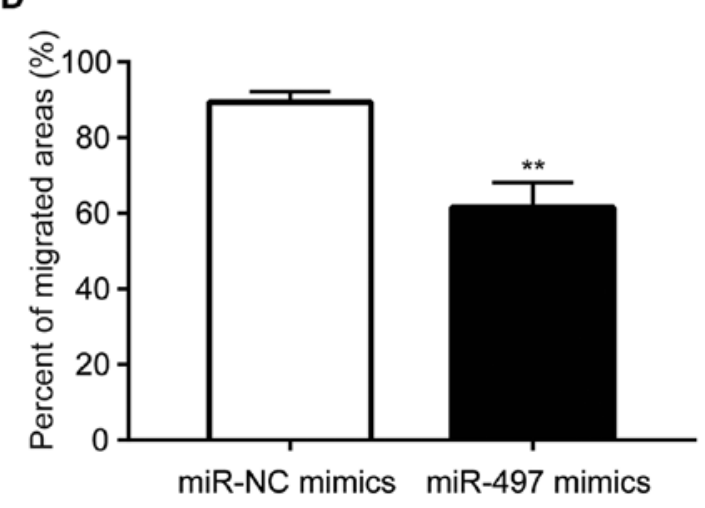

Figure 2. Overexpression of miR-497 inhibits non-small cell lung cancer cell proliferation and migration. (A) Transfection with miR-497 mimics elevated miR-497 levels in A549 cells. (B) Cell proliferation was inhibited following miR-497 overexpression in A549 cells. (C) Representative images of migrated A549 cells transfected with miR-NC mimics or miR-497 mimics. Magnification, x100. (D) Quantitative analysis of cell migration area. The experiment was repeated $\geq 3$ times. ${ }^{* *} \mathrm{P}<0.01,{ }^{* * * *} \mathrm{P}<0.0001$ vs. miR-NC. miR, microRNA; NC, negative control. 
A

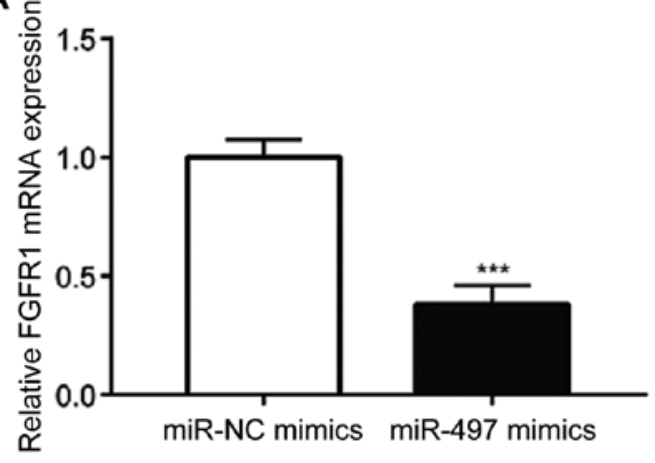

B

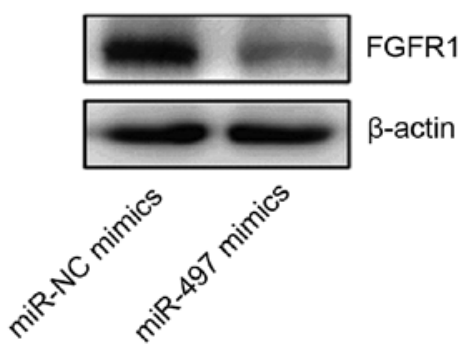

C

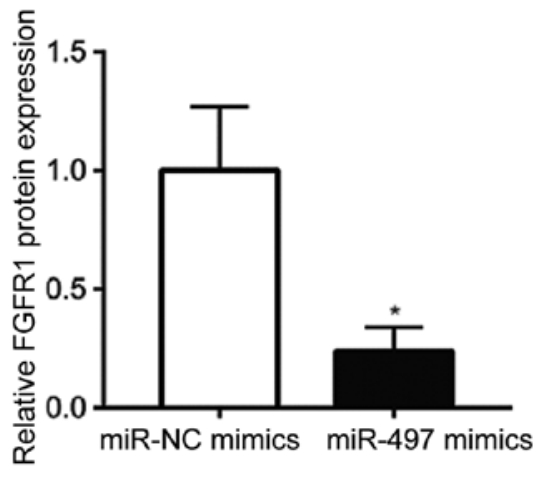

E
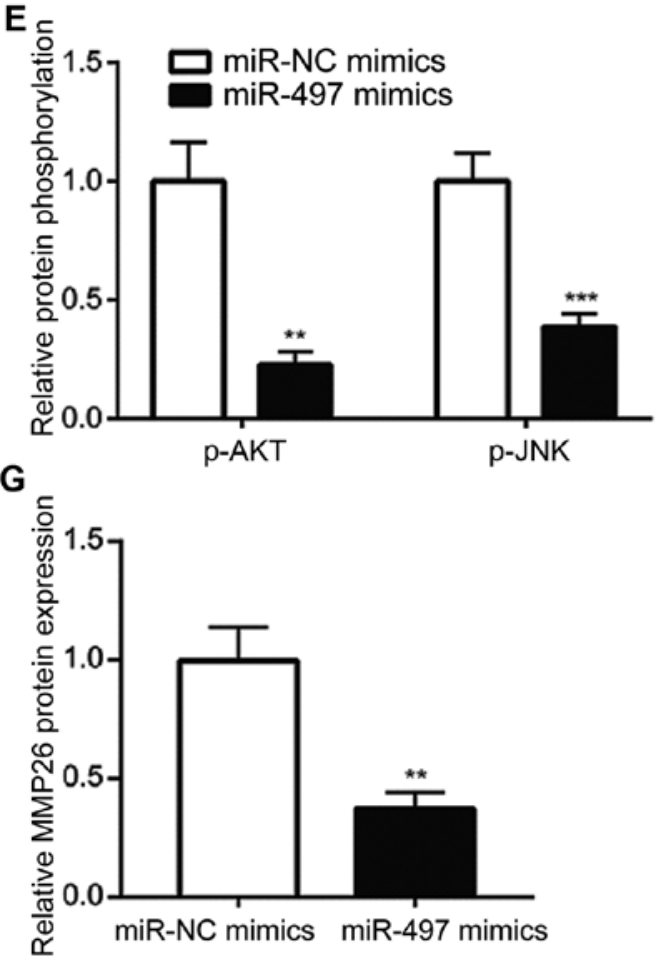

D

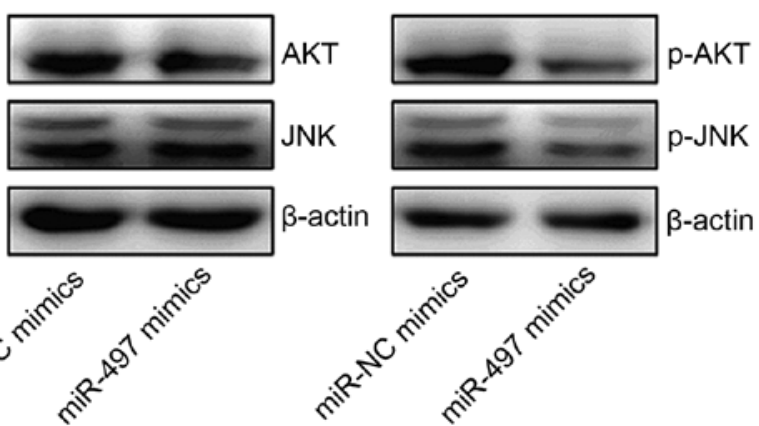

F
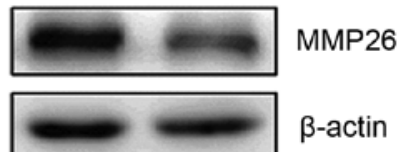

Figure 3. miR-497 overexpression suppresses FGFR1 expression and inactivated its downstream signaling pathways in A549 cells. (A) Transfection of miR-497 mimics decreased FGFR1 mRNA levels in A549 cells. (B) miR-497 mimics decreased FGFR1 protein levels in A549 cells. (C) Semi-quantitative analysis of FGFR1 protein levels. (D) miR-497 mimics reduced p-AKT and p-JNK levels in A549 cells. (E) Semi-quantitative analysis of p-AKT and p-JNK protein levels. (F) MMP26 protein levels were decreased in A549 cells following miR-497 overexpression. (G) Semi-quantitative analysis of MMP26 protein levels. The experiment was repeated $\geq 3$ times. ${ }^{*} \mathrm{P}<0.05,{ }^{* *} \mathrm{P}<0.01,{ }^{* * *} \mathrm{P}<0.0001$ vs. miR-NC. FGFR1, fibroblast growth factor receptor 1 ; miR, microRNA; NC, negative control; AKT, protein kinase B; JNK, c-Jun N-terminal kinase; p-, phosphorylated; MMP26, matrix metallopeptidase 26.

Therefore, these results indicate that miR-497 may function as a tumor suppressor in NSCLC via regulation of FGFR1 and the downstream targets of FGFR1.
miR-497 binds to the 3'-UTR of FGFR1 mRNA. Using TargetScan software, predicted complementary binding sites between miR-497 and the FGFR1 3'-UTR sequence were 


\section{A $874:$ 5' cucAAUAAAAAU---UGGAGCUg 3' FGFR1 Mut

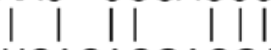 \\ 3' uguUUGGUGUCACACGACGAC 5' hsa-miR-497 \\ | | | || || || \\ 874 : 5' cucAAUAAAAAU-UGCUGCUg 3' FGFR1 WT}

B

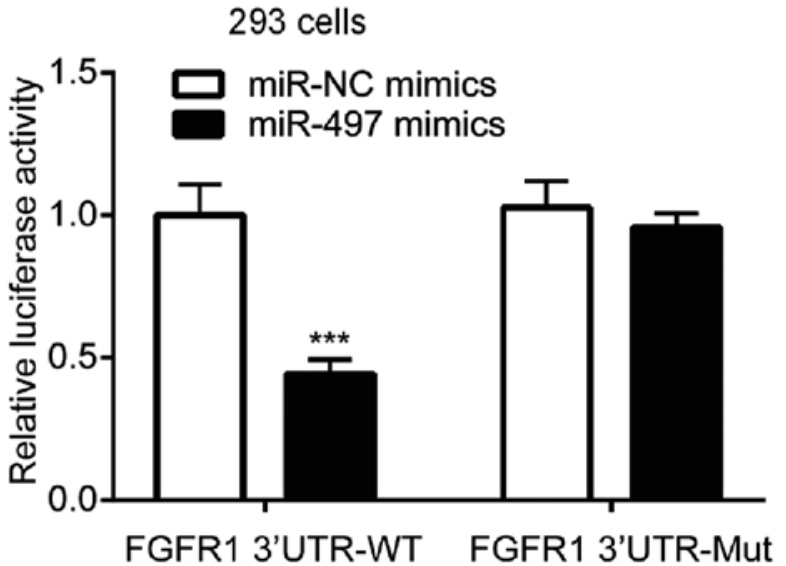

C

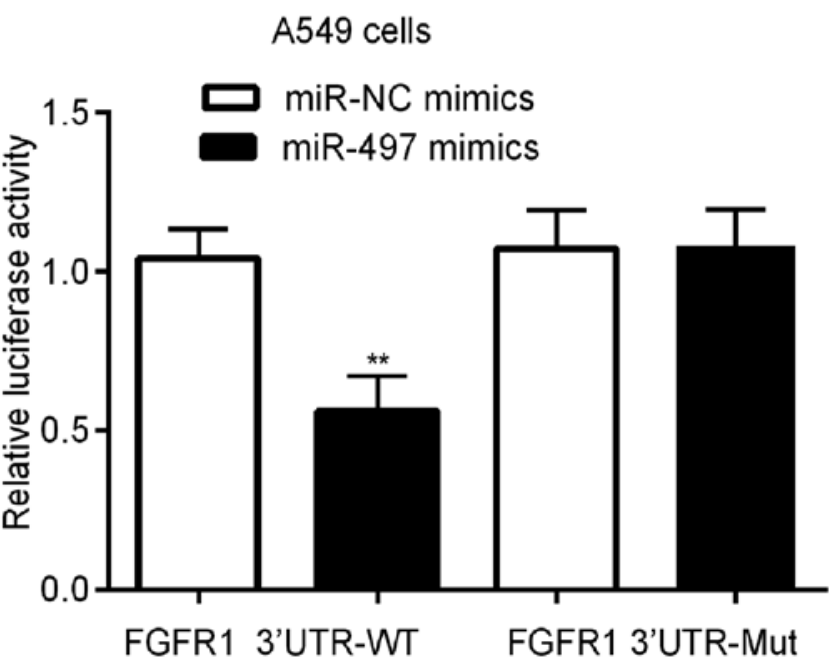

Figure 4. miR-497 directly binds the 3'-UTR of FGFR1 mRNA. (A) Alignment of the miR-497 and the FGFR1 3'-UTR sequences indicates a complementary binding site. In (B) 293 cells and (C) A549 cells, dual luciferase reporter assay analysis indicates that miRNA-497 reduces the relative luciferase activity of wild-type, not mutant, FGFR1 3'-UTR mRNA sequences. The experiment was repeated $\geq 3$ times. ${ }^{* *} \mathrm{P}<0.01,{ }^{* * *} \mathrm{P}<0.0001$. FGFR1, fibroblast growth factor receptor 1; Mut, mutant; WT, wild-type; miR, microRNA; NC, negative control; UTR, untranslated region.

identified, indicating that miR-497 may bind to 3'-UTR of FGFR1 mRNA (Fig. 4A). The subsequent dual luciferase reporter assay analysis demonstrated that transfection with miR-497 mimics repressed luciferase activity in 239 and A549 cells transfected with pGL3-FGFR1 3'-UTR-WT vector, but not the pGL3-FGFR1-Mutant vector containing mutations in two potential binding nucleotides (Fig. 4B and C).

FGFRl is involved in miR-497 mimic-induced arrest of cell growth and migration in A549 cells. To elucidate the role of FGFR1 in the miR-497-mediated inhibition of cancer progression, a pcDNA3-FGFR1 plasmid was used to overexpress FGFR1 in A549 cells. As shown in Fig. 5A and B, transfection of pcDNA3-FGFR1 elevated FGFR1 protein levels in A549 cells. Overexpression of FGFR1 significantly reversed cell proliferation inhibition induced by miR-497 mimics in A549 cells (Fig. 5C). Furthermore, miR-497 mimics-induced inhibition of cell migration was rescued by transfection with
pcDNA3-FGFR1 (Fig. 5D and E). Collectively, these data demonstrate that miR-497 inhibited NSCLC progression via the regulation of FGFR1.

\section{Discussion}

Overexpression of FGFR1 is frequently observed in lung cancer and is considered to be a biomarker of response to FGFR-tyrosine kinase inhibitor (TKI) therapy in patients with lung cancer $(28,29)$. FGFR1 gene amplification is known to be an underlying cause of increased FGFR1 expression in lung cancer, and gene copy number (GCN) is used to predict patient response to FGFR1-TKI in the clinic $(30,31)$. However, a recent study reported that FGFR1 mRNA levels may be an improved indicator of patient response to FGFR-TKI therapy compared with GCN, suggesting that gene amplification may sufficiently explain the increased FGFR1 expression levels in lung cancer (32). Indeed, a subsequent study demonstrated that FGFR1 expression was also 
A

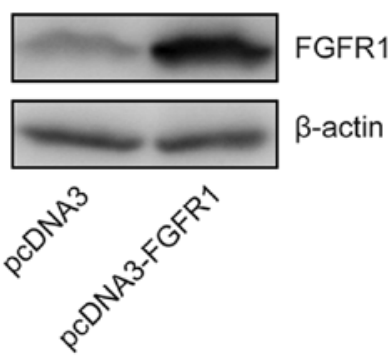

B

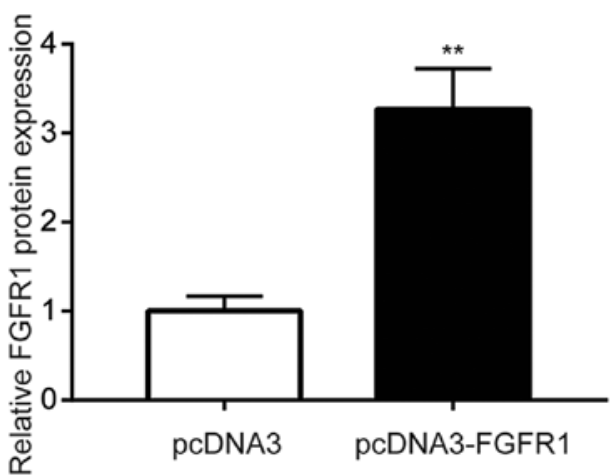

C

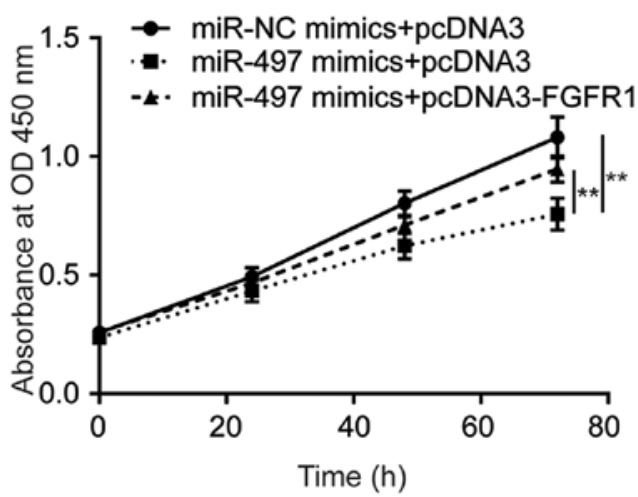

E

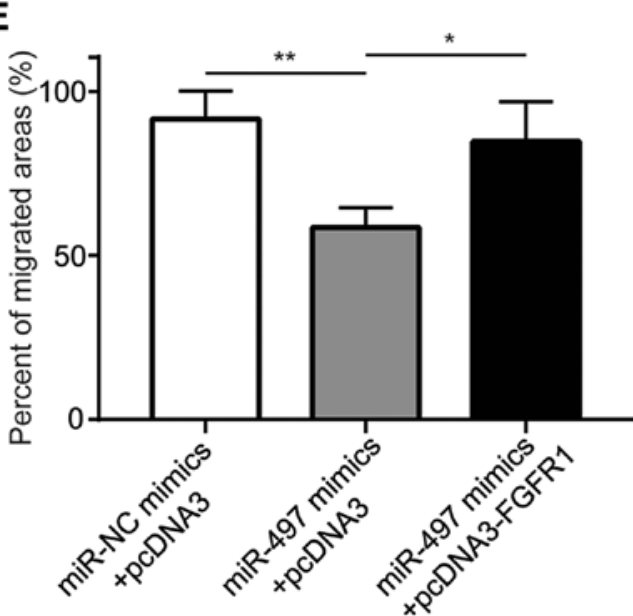

D

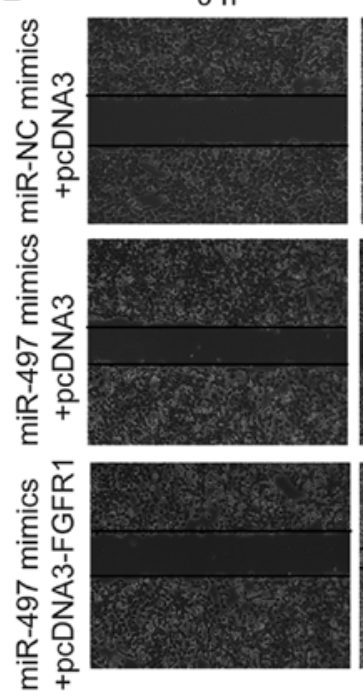

$30 \mathrm{~h}$
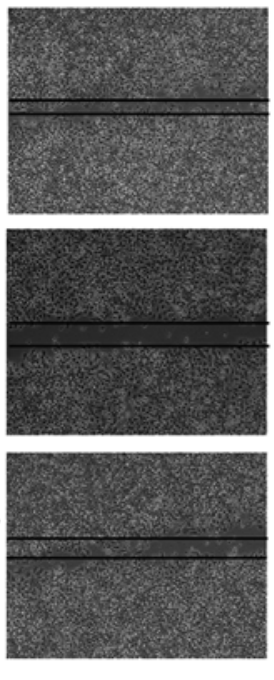

Figure 5. Overexpression of FGFR1 reverses the miR-497 mimics-induced inhibition of cell proliferation and migration in A549 cells. (A) Transfection of pcDNA3-FGFR1 elevates FGFR1 protein expression in A549 cells. (B) Semi-quantification of FGFR1 protein expression. ${ }^{* *}$ P<0.01 vs. pcDNA3. (C) miR-497 mimics reduces the proliferative ability of A549 cells, which is reversed by FGFR1 overexpression. (D) miR-497 mimics reduces the migration of A549 cells, which was reversed by FGFR1 overexpression. Magnification, x100. (E) Quantification of cell migration. * $\mathrm{P}<0.05 ;{ }^{* * *} \mathrm{P}<0.01$. The experiment was repeated $\geq 3$ times. FGFR1, fibroblast growth factor receptor 1; miR, microRNA; NC, negative control.

regulated by miR-198 in lung cancer (33). In the present study, a negative correlation between miR-497 and FGFR1 mRNA levels in NSCLC tumor tissues was observed. In A549 cells, RT-qPCR and western blot analyses demonstrated that miR-497 repressed FGFR1 at the mRNA and protein expression levels. In addition, the PI3K-AKT and JNK signaling pathways, which are two downstream signaling pathways of FGFR1, were inactivated following miR-497 overexpression. The expression of the MMP26 oncogene was also negatively regulated by miR-497 in A549 cells. The present study also predicted and validated FGFR1 as a target gene of miR-497 in A549 cells.
Previous studies have demonstrated that miR-497 expression is downregulated in NSCLC, and that miR-497 targets multiple oncogenes to promote NSCLC progression $(34,35)$. In the present study, overexpression of miR-497 inhibited the proliferation and migration of NSCLC cells, which is consistent with previous reports $(36,37)$. Notably, overexpression of FGFR1 reversed the miR-497 mimic-induced arrest of cell growth and migration in A549 cells, suggesting that miR-497 may primarily rely on the regulation of FGFR1 to inhibit NSCLC progression. In addition, there are a number of studies on the function of miR-497 in lung cancer by targeting other mRNA targets. For instance, miR-497 
was reported to inhibit the epithelial-mesenchymal transition of NSCLC by targeting Metadherin (38), miR-497 was discovered to inhibit the tumor growth of NSCLC by targeting YAP1 (34) and miR-497 was found to inhibit cell growth and invasion of NSCLC by targeting VEGF-A (36).

In conclusion, the results of the current study suggest that miR-497 may function as a tumor suppressor in NSCLC via regulation of FGFR1. In NSCLC cells, miR-497 binds directly to the 3'-UTR of FGFR1 mRNA, leading to reduction in FGFR1 protein levels, inactivation of the PI3K-AKT and JNK signaling pathways and the downregulation of MMP26 expression. These findings enrich the current understanding of miR-497 and FGFR1 in mediating NSCLC progression, and implicate miR-497 as a putative therapeutic target for patients with NSCLC.

\section{Acknowledgements}

Not applicable.

\section{Funding}

No funding was received.

\section{Availability of data and materials}

The datasets used and/or analyzed during the present study are available from the corresponding author on reasonable request.

\section{Authors' contributions}

The present study was designed by HL. The experiments were carried out by QH, XD, DZ, BG and WX. The data were analyzed by $\mathrm{QH}, \mathrm{HL}$ and XD. The manuscript was written by HL, and all authors approved the final version of the manuscript.

\section{Ethics approval and consent to participate}

The present study was approved by the Ethics Committee of Jingzhou First People's Hospital. Written informed consent was provided by the patients or their family.

\section{Patient consent for publication}

The present study received consent for publication from each patient.

\section{Competing interests}

The authors declare that they have no competing interests.

\section{References}

1. Torre LA, Bray F, Siegel RL, Ferlay J, Lortet-Tieulent J and Jemal A: Global cancer statistics, 2012. CA Cancer J Clin 65: 87-108, 2015.

2. Marshall AL and Christiani DC: Genetic susceptibility to lung cancer-light at the end of the tunnel? Carcinogenesis 34: 487-502, 2013.

3. Siegel R, Naishadham D and Jemal A: Cancer statistics, 2013. CA Cancer J Clin 63: 11-30, 2013.
4. Wistuba II, Gelovani JG, Jacoby JJ, Davis SE and Herbst RS: Methodological and practical challenges for personalized cancer therapies. Nat Rev Clin Oncol 8: 135-141, 2011.

5. Tiong KH, Mah LY and Leong CO: Functional roles of fibroblast growth factor receptors (FGFRs) signaling in human cancers. Apoptosis 18: 1447-1468, 2013.

6. Monaco SE, Rodriguez EF, Mahaffey AL and Dacic S: FGFR1 amplification in squamous cell carcinoma of the lung with correlation of primary and metastatic tumor status. Am J Clin Pathol 145: 55-61, 2016.

7. Young RJ, Lim AM, Angel C, Collins M, Deb S, Corry J, Wiesenfeld D, Kleid S, Sigston E, Lyons B, et al: Frequency of fibroblast growth factor receptor 1 gene amplification in oral tongue squamous cell carcinomas and associations with clinical features and patient outcome. Oral Oncol 49: 576-581, 2013.

8. Ishizuka T, Tanabe C, Sakamoto H, Aoyagi K, Maekawa M, Matsukura N, Tokunaga A, Tajiri T, Yoshida T, Terada M and Sasaki H: Gene amplification profiling of esophageal squamous cell carcinomas by DNA array CGH. Biochem Biophys Res Commun 296: 152-155, 2002

9. Gru AA and Allred DC: FGFR1 amplification and the progression of non-invasive to invasive breast cancer. Breast Cancer Res 14: 116, 2012.

10. Kim HR, Kim DJ, Kang DR, Lee JG, Lim SM, Lee CY, Rha SY, Bae MK, Lee YJ, Kim SH, et al: Fibroblast growth factor receptor 1 gene amplification is associated with poor survival and cigarette smoking dosage in patients with resected squamous cell lung cancer. J Clin Oncol 31: 731-737, 2013.

11. Wang Y, Cai Y, Ji J, Liu Z, Zhao C, Zhao Y, Wei T, Shen X, Zhang X, Li X and Liang G: Discovery and identification of new non-ATP competitive FGFR1 inhibitors with therapeutic potential on non-small-cell lung cancer. Cancer Lett 344: 82-89, 2014.

12. Pu D, Liu J, Li Z, Zhu J and Hou M: Fibroblast growth factor receptor 1 (FGFR1), partly related to vascular endothelial growth factor receptor 2 (VEGFR2) and microvessel density, is an independent prognostic factor for non-small cell lung cancer. Med Sci Monit 23: 247-257, 2017.

13. Bartel DP: MicroRNAs: Genomics, biogenesis, mechanism, and function. Cell 116: 281-297, 2004.

14. Kawahara Y: Human diseases caused by germline and somatic abnormalities in microRNA and microRNA-related genes. Congenit Anom (Kyoto) 54: 12-21, 2014.

15. Iorio MV and Croce CM: MicroRNAs in cancer: Small molecules with a huge impact. J Clin Oncol 27: 5848-5856, 2009.

16. Kasinski AL and Slack FJ: miRNA-34 prevents cancer initiation and progression in a therapeutically resistant K-ras and p53-induced mouse model of lung adenocarcinoma. Cancer Res 72: 5576-5587, 2012.

17. Fortunato O, Boeri M, Moro M, Verri C, Mensah M, Conte D, Caleca L, Roz L, Pastorino U and Sozzi G: Mir-660 is downregulated in lung cancer patients and its replacement inhibits lung tumorigenesis by targeting MDM2-p53 interaction. Cell Death Dis 5: e1564, 2014

18. Peng Y, Dai Y, Hitchcock C, Yang X, Kassis ES, Liu L, Luo Z, Sun HL, Cui R, Wei H, et al: Insulin growth factor signaling is regulated by microRNA-486, an underexpressed microRNA in lung cancer. Proc Natl Acad Sci USA 110: 15043-15048, 2013.

19. Wang J, Li J, Wang X, Zheng C and Ma W: Downregulation of microRNA-214 and overexpression of FGFR-1 contribute to hepatocellular carcinoma metastasis. Biochem Biophys Res Commun 439: 47-53, 2013.

20. Jiang H, Qu L, Wang Y, Cong J, Wang W and Yang X: miR-99a promotes proliferation targeting FGFR3 in human epithelial ovarian cancer cells. Biomed Pharmacother 68: 163-169, 2014.

21. Nishijima N, Seike M, Soeno C, Chiba M, Miyanaga A, Noro R, Sugano T, Matsumoto M, Kubota K and Gemma A: miR-200/ZEB axis regulates sensitivity to nintedanib in non-small cell lung cancer cells. Int J Oncol 48: 937-944, 2016.

22. Chen QY, Jiao DM, Yan L, Wu YQ, Hu HZ, Song J, Yan J, Wu LJ, $\mathrm{Xu}$ LQ and Shi JG: Comprehensive gene and microRNA expression profiling reveals miR-206 inhibits MET in lung cancer metastasis. Mol Biosyst 11: 2290-2302, 2015.

23. Wang R, Chen XF and Shu YQ: Prediction of non-small cell lung cancer metastasis-associated microRNAs using bioinformatics. Am J Cancer Res 5: 32-51, 2014.

24. Livak KJ and Schmittgen TD: Analysis of relative gene expression data using real-time quantitative PCR and the 2(-Delta Delta C(T)) method. Methods 25: 402-408, 2001. 
25. Hu F, Liu H, Xie X, Mei J and Wang M: Activated cdc42-associated kinase is up-regulated in non-small-cell lung cancer and necessary for FGFR-mediated AKT activation. Mol Carcinog 55: 853-863, 2016.

26. Jung SY, Yi JY, Kim MH, Song KH, Kang SM, Ahn J, Hwang SG, Nam KY and Song JY: IM-412 inhibits the invasion of human breast carcinoma cells by blocking FGFR-mediated signaling. Oncol Rep 34: 2731-2737, 2015.

27. Zhao D, Lu Y, Yang C, Zhou X and Xu Z: Activation of FGF receptor signaling promotes invasion of non-small-cell lung cancer. Tumour Biol 36: 3637-3642, 2015.

28. Theelen WS, Mittempergher L, Willems SM, Bosma AJ, Peters DD, van der Noort V, Japenga EJ, Peeters T, Koole K, Šuštić $\mathrm{T}$, et al: FGFR1, 2 and 3 protein overexpression and molecular aberrations of FGFR 3 in early stage non-small cell lung cancer. J Pathol Clin Res 2: 223-233, 2016.

29. Göke A, Franzen A, Menon R, Goltz D, Kirsten R, Boehm D, Vogel W, Scheble V, Ellinger J, Gerigk U, et al: Rationale for treatment of metastatic squamous cell carcinoma of the lung using fibroblast growth factor receptor inhibitors. Chest 142 : 1020-1026, 2012.

30. Tran TN, Selinger CI, Kohonen-Corish MR, McCaughan BC, Kennedy CW, O'Toole SA and Cooper WA: Fibroblast growth factor receptor 1 (FGFR1) copy number is an independent prognostic factor in non-small cell lung cancer. Lung Cancer 81: 462-467, 2013

31. Dutt A, Ramos AH, Hammerman PS, Mermel C, Cho J, Sharifnia T, Chande A, Tanaka KE, Stransky N, Greulich H, et al: Inhibitor-sensitive FGFR1 amplification in human non-small cell lung cancer. PLoS One 6: e20351, 2011.
32. Wynes MW, Hinz TK, Gao D, Martini M, Marek LA, Ware KE, Edwards MG, Böhm D, Perner S, Helfrich BA, et al: FGFR1 mRNA and protein expression, not gene copy number, predict FGFR TKI sensitivity across all lung cancer histologies. Clin Cancer Res 20: 3299-3309, 2014

33. Yang J,Zhao H, Xin Y and Fan L: MicroRNA-198 inhibits proliferation and induces apoptosis of lung cancer cells via targeting FGFR1. J Cell Biochem 115: 987-995, 2014

34. Huang C, Ma R, Yue J, Li N, Li Z and Qi D: MiR-497 suppresses YAP1 and inhibits tumor growth in non-small cell lung cancer. Cell Physiol Biochem 37: 342-352, 2015.

35. Zhao WY, Wang Y, An ZJ, Shi CG, Zhu GA, Wang B, Lu MY, Pan CK and Chen P: Downregulation of miR-497 promotes tumor growth and angiogenesis by targeting HDGF in non-small cell lung cancer. Biochem Biophys Res Commun 435: 466-471, 2013.

36. Gu A, Lu J, Wang W, Shi C, Han B and Yao M: Role of miR-497 in VEGF-A-mediated cancer cell growth and invasion in non-small cell lung cancer. Int J Biochem Cell Biol 70: 118-125, 2016.

37. Han Z, Zhang Y, Yang Q, Liu B, Wu J, Zhang Y, Yang C and Jiang Y: miR-497 and miR-34a retard lung cancer growth by co-inhibiting cyclin E1 (CCNE1). Oncotarget 6: 13149-13163, 2015.

38. Yin Q, Han Y,Zhu D, Li Z, Shan S, Jin W,Lu Q and Ren T: miR-145 and miR-497 suppress TGF- $\beta$-induced epithelial-mesenchymal transition of non-small cell lung cancer by targeting MTDH. Cancer Cell Int 18: 105, 2018. 